\title{
LE RISQUE DE CONTIGUITE DANS \\ L'ASSURANCE INCENDIE DES BATIMENTS
}

\author{
Paul Johansen \\ Copenhague, Danemark
}

I

Le calcul de la prime de l'assurance Incendie des bâtiments se trouve compliqué par le fait qu'il doit tenir compte, non seulement du bâtiment assuré, mais également des immeubles voisins.

Si deux bâtiments sont suffisamment éloignés, le risque qu'un incendie se propage de l'un à l'autre est minime, mais le risque de contiguité, et par conséquent d'incendie total, est d'autant plus grand que leur distance est plus faible.

La mesure dans laquelle le risque de contiguité doit être retenu pour la tarification est un des problèmes les plus importants que l'Actuaire a à résoudre en assurance Incendie.

Pour qu'un incendie puisse s'étendre à d'autres bâtiments, le feu doit embrasser d'abord les éléments extérieurs du bâtiment assuré, c'est-à-dire les murs, la cheminée, la toiture ou les constructions en bois attenantes. Nous appellerons de tels incendies: incendies extérieurs par opposition à incendies intérieurs qui n'embrassent que le contenu du bâtiment et les aménagements intérieurs.

II

Par risque propre d'un bâtiment nous entendons l'indemnité moyenne annuelle de sinistre, sous la condition que ce bâtiment ne soit pas exposé au risque de contiguité d'autres immeubles.

Le risque propre se divise en une fraction qui concerne les incendies intérieurs et une autre fraction concernant les incendies extérieurs,

$$
R_{p}=R_{i}+R_{\epsilon}
$$

Le deuxième terme $\mathrm{R}_{e}$ peut être exprimé par le produit de la probabilité d'un incendie extérieur et de l'indemnité moyenne de cet incendie, soit 


$$
R_{e}=p_{e} \cdot M_{e}
$$

Les éléments de ce risque propre peuvent être observés pour différents groupes de risques, c'est-à-dire chaque groupe comprenant des bâtiments d'un même genre, par exemple en ce qui concerne leur construction, leur dimension ou leur destination.

Les incendies ayant leur origine dans d'autres bâtiments doivent être exclus du calcul du risque propre.

Si nous considérons simultanément plusieurs bâtiments $A, B$, etc... nous désignerons leur risque propre respectif par les indices $A, B$, etc...

\section{III}

Le risque Incendie total est constitué par la somme du risque propre du bâtiment et le risque de contiguité, soit:

$$
R_{t}=R_{p}+R_{c}
$$

Considérons le bâtiment $A$ et l'autre bâtiment voisin $B$. Nous désignerons par $M_{c}$ le sinistre moyen causé par la contiguïté et par $p(B, A)$ la probabilité de contiguité, compte tenu de la construction des deux bâtiments et de la distance, $d$, qui les sépare. On a évidemment:

$$
R_{t}^{A}=R_{i}^{A}+p_{e}^{A} \cdot M_{e}^{A}+p_{e}^{B} \cdot M_{c}^{A} \cdot p(B, A)
$$

Pour ne pas trop compliquer l'exposé, nous ne retiendrons que la possibilité de contiguïté d'un seul bâtiment voisin.

D'une manière analogue, on peut écrire pour le bâtiment $B$ :

$$
R_{t}^{B}=R_{i}^{B}+p_{e}^{B} \cdot M_{e}^{B}+p_{e}^{A} \cdot M_{c}^{B} \cdot p(A, B)
$$

\section{IV}

Pour illustrer l'ordre de grandeur de ces quantités, nous donnerons des valeurs numériques mesurées pour des risques ruraux danois. La valeur de cette statistique est restreinte s'agissant seulement d'environ 2.000 incendies extérieurs.

Les fermes danoises, dans leur majorité, sont construites en briques, couvertes d'ardoises, de tuiles ou d'autres matériaux durs. Cependant, quelques bâtiments anciens sont encore couverts de paille, de roseau ou de chaume. 
Prenons deux classes de construction:

la classe $A$ : couverture dure

la classe $B$ : couverture de chaume.

Supposons que les sommes assurées sur les bâtiments considérés soient égales à t'unité.

Pour le risque propre nous pouvons fixer les valeurs comme suit:

$$
\begin{aligned}
& R_{i}^{A}=0,3 \%, p_{e}^{A}=0,5 \%, M_{e}^{A}=0,75 R_{p}^{A}=0,68 \% \\
& R_{i}^{B}=0,5 \%, p_{e}^{B}=2,5 \%, M_{e}^{B}=0,87 R_{p}^{B}=2,68 \%
\end{aligned}
$$

Dans les quatre situations de contiguité, nous avons trouvé les valeurs suivantes pour $p$ et $M_{c}$ selon la distance $d$ qui sépare les deux bâtiments. Dans le groupe $d \angle 1$ mètre on trouve surtout des bâtiments qui se touchent.

\begin{tabular}{|c|c|c|c|c|c|}
\hline$d$ & $p(A, A)$ & $M_{c}^{A}$ & $R_{c}^{A}$ & $R_{t}^{A}$ & $R_{t} / R_{p}$ \\
\hline O-I m. & 0,76 & 0,59 & $0,22 \%$ & $0,90 \%$ & 1,32 \\
\hline $\mathrm{I}-5 \mathrm{~m}$. & $0,2 \mathrm{I}$ & 0,14 & O,OI $\%$ & $0,69 \%$ & $\mathrm{I}, \mathrm{OI}$ \\
\hline $5-30 \mathrm{~m}$ & 0,09 & 0,15 & o,or $\%$ & $0,69 \%$ & $\mathrm{I}, \mathrm{OI}$ \\
\hline
\end{tabular}

Contiguité de toit dur à toit dur, $R_{P}^{A}=0,68 \%$

Contiguité de toit dur à toit de chaume, $\mathrm{R}_{p}^{B}=2,68 \%$

\begin{tabular}{ccccccc}
\hline$d$ & $p(A, B)$ & $M_{c}^{B}$ & $R_{c}^{B}$ & $R_{t}^{B}$ & $R_{t} / R_{p}$ \\
\hline o-I & m. & 0,79 & 0,96 & $0,38 \%$ & $3,06 \%$ & $\mathrm{I}, \mathrm{I} 4$ \\
I-5 m. & 0,38 & 0,35 & $0,07 \%$ & $2,75 \%$ & $\mathrm{I}, 03$ \\
5-30 m. & 0,18 & 0,65 & $0,06 \%$ & $2,74 \%$ & $\mathrm{I}, 02$
\end{tabular}

\begin{tabular}{|c|c|c|c|c|c|}
\hline$d$ & $p(B, A)$ & $M_{c}^{A}$ & $R_{c}^{A}$ & $R_{t}^{A}$ & $R_{t} / R_{p}$ \\
\hline o-I m. & 0,72 & 0,53 & $0,95 \%$ & $I, 63 \%$ & 2,40 \\
\hline I-5 m. & 0,54 & 0,25 & $0,07 \%$ & $1,02 \%$ & 1,50 \\
\hline $5-30 \mathrm{~m}$ & 0,39 & 0,15 & $0,06 \%$ & $0,83 \%$ & 1,22 \\
\hline
\end{tabular}

Contiguité de toit de chaume à toit dur, $\mathrm{R}_{p}^{A}=0,68 \%$

Contiguité de toit de chaume à toit de chaume, $\mathrm{R}_{p}^{B}=2,68 \%$

\begin{tabular}{ccccccc}
\hline$d$ & $p(B, B)$ & $M_{c}^{B}$ & $R_{c}^{B}$ & $R_{t}^{B}$ & $R_{t} / R_{p}$ \\
\hline o-I & m. & $0,9 \mathrm{I}$ & 0,85 & $\mathrm{I}, 93 \%$ & $4,6 \mathrm{I} \%$ & $\mathrm{I}, 72$ \\
$\mathrm{r}-5$ & $\mathrm{~m}$. & 0,74 & 0,67 & $\mathrm{I}, 24 \%$ & $3,92 \%$ & $\mathrm{1}, 46$ \\
$5-30$ & $\mathrm{~m}$. & 0,28 & 0,76 & $0,53 \%$ & $3,2 \mathrm{I} \%$ & $\mathrm{I}, 2 \mathrm{0}$ \\
\hline
\end{tabular}


On voit que l'on peut négliger le risque de contiguïté des bâtiments voisins, tous deux avec couverture dure, quand ils sont séparés même par une faible distance.

Des bâtiments voisins, couverts en chaume, très proches l'un de l'autre, entraînent une augmentation de prime propre d'environ $50 \%$, et des bâtiments de chaume, un peu plus éloignés, une augmentation d'environ $20 \%$.

\section{$\mathrm{V}$}

Si par une même police on assure deux bâtiments pour une somme égale, les primes totales doivent être fixées comme suit:

\begin{tabular}{|c|c|c|c|c|c|c|}
\hline & \multicolumn{2}{|c|}{$\begin{array}{c}\text { Deux bâtiments } \\
\text { de couverture } \\
\text { dure }\end{array}$} & \multicolumn{2}{|c|}{$\begin{array}{l}\text { l'un couverture } \\
\text { dure } \\
\text { l'autre de chaume }\end{array}$} & \multicolumn{2}{|c|}{$\begin{array}{c}\text { Deux bâtiments } \\
\text { de chaume }\end{array}$} \\
\hline & \multicolumn{2}{|c|}{$R_{p}=2.0,68 \%$} & \multicolumn{2}{|c|}{$R_{p}=0,68 \%+$} & \multicolumn{2}{|c|}{$R_{p}=2.2,68 \%$} \\
\hline$d$ & $R_{t}$ & $R_{t} / R_{p}$ & $R_{t}$ & $R_{t} / R_{p}$ & $R_{t}$ & $R_{t} / R_{p}$ \\
\hline $\mathrm{o}-\mathrm{I} \mathrm{m}$. & $2.0,90 \%$ & $r, 32$ & $1,63+3,06 \%$ & $I, 4^{\circ}$ & $2.4,6 \mathrm{r} \%$ & $\mathrm{I}, 72$ \\
\hline $\mathrm{I}-5 \mathrm{~m}$ & $2.0,69 \%$ & $I, O I$ & $1,02+2,75 \%$ & $I, I 2$ & $2.3,91 \%$ & 1,46 \\
\hline $5-30 \mathrm{~m}$ & $2.0,69 \%$ & $\mathrm{I}, \mathrm{OI}$ & $0,83+2,74 \%$ & I,06 & $2.3,21 \%$ & $\mathrm{I}, 20$ \\
\hline
\end{tabular}

Le tarif doit prescrire une distance plus élevée pour deux bâtiments couverts en chaume que pour un bâtiment couvert en chaume et un autre couvert en dur. 